Polymer Journal, Vol. 10, No. 1, pp 37-44 (1978)

\title{
Formation of Polyelectrolyte Complex of Heparin with Aminoacetalized Poly(vinyl alcohol)
}

\author{
Masanori HaRA and Akio Nakajima \\ Department of Polymer Chemistry, Kyoto University, \\ Kyoto 606, Japan.
}

(Received June 30, 1977)

\begin{abstract}
The formation of a polyelectrolyte complex between heparin (Hep) and aminoacetalized poly(vinyl alcohol) (PVAA) in aqueous media has been studied by measurements of turbidity, metachromasy, and induced circular dichroism. Three results were noted: (a) the Hep-PVAA complex in aqueous system is either in a state of liquid-solid equilibrium, or a homogeneous solution, according to the mixing conditions; (b) the reaction between Hep and PVAA was not perfectly stoichiometric; (c) the ordered structure of Hep was disordered by the formation of complex at low $\mathrm{pH}$.

KEY WORDS Polyelectrolyte Complex/Heparin / Aminoacetalized Poly(vinyl alcohol) / Soluble Complex / Turbidity / Metachromasy /

Circular Dichroism /
\end{abstract}

When a polycation is mixed with a polyanion in an aqueous medium, a reaction between the oppositely charged residues takes place and a polyelectrolyte complex is formed. According to the mixing conditions, the reaction mixture is considered to exist in one of three states: (1) liquid-liquid equilibrium state (so-called complex coacervation); (2) liquid-solid equilibrium state (owing to insoluble complex); (3) homogeneous solution (owing to soluble complex). The complex particles in each phase may be in the form of a neutral polysalt resulting from the stoichiometric reaction, or of a polysalt with excess charges.

The stoichiometry of the reaction between polyelectrolytes with opposite signs has been investigated by several workers. ${ }^{1-4}$ The stoichiometric reaction was observed for the system of two polyelectrolytes with flexible backbone chains each carrying strong acid residues and strong base residues, respectively, while the nonstoichiometric reaction was observed for the system of polyelectrolytes with a rigid backbone chain..$^{3,4}$ These results are attributed to steric factors: the facility or difficulty of changing the distance between charges owing to the chain flexibility or rigidity.
Little is known about the structure of the complex in solution. The structure of the complex containing polypeptides as one or both components has come under investigation using circular dichroism (CD) spectroscopy, recently. ${ }^{5-13}$ But the structure of the complexes composed of polymers other than polypeptides has not been accounted for in detail.

In this paper, we are concerned with the formation of the polyelectrolyte complex composed of heparin (Hep) and aminoacetalized poly(vinyl alcohol) (PVAA), and we discuss the state of the complex in an aqueous system, the stoichiometry of the reaction, and the structure of Hep in the complex. Hep is a mucopolysaccharide having such important physiological activities as anticoagurant activity and lypolytic activity, and has been investigated by many workers. Though the primary and secondary structures are not perfectly known, Hep is composed of $\alpha$-1,4-glycosidic linkage and regarded as a Gaussian coil having a locally helical (ordered) structure in solution. ${ }^{14}$

\section{EXPERIMENTAL}

\section{Materials}

Heparin-Na (Lot: M3E7945) was purchased 


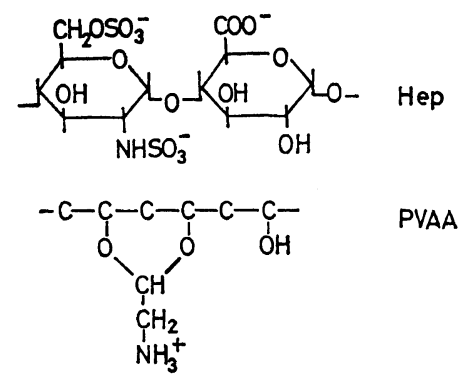

Figure 1. Chemical structures of Hep and PVAA.

Table I. Summary of the characterization of polyelectrolytes used

\begin{tabular}{lcccc}
\hline Polymer & $M$ & $\begin{array}{l}\text { Ionizable } \\
\text { group }\end{array}$ & $\mathrm{p} K_{\mathrm{a}}$ & $\begin{array}{c}\text { Remarks on } \\
\text { ionizable groups }\end{array}$ \\
\hline Hep & 9000 & $\begin{array}{l}\mathrm{SO}_{3} \mathrm{H} \\
\mathrm{COOH}\end{array}$ & 5.05 & $\frac{\mathrm{SO}_{3} \mathrm{H}}{\mathrm{COOH}}=2.0$ \\
\hline PVAA & 44000 & $\mathrm{NH}_{2}$ & 8.10 & $7.5 \mathrm{NH}_{2} / 100$ residue
\end{tabular}

from Nakarai Chemicals Co., Kyoto, and used without further purification. Poly(vinyl alcohol) (PVA) obtained from Kuraray Co. was fractionated with a 1-propanol-water mixture to remove the high and low molecular weight fractions. Aminoacetalized poly(vinyl alcohol) (PVAA) was obtained by reacting PVA with aminoacetal in the presence of $\mathrm{HCl}$ at $70^{\circ} \mathrm{C}$. PVAA was purified by dialysis and converted into a basic polymer by passing it through an ion-exchange resin. Acridine orange was purified by the method reported by Myhr and Foss. ${ }^{15}$ The chemical structures of these polymers are shown in Figure 1.

\section{Characterization of Polymer Samples}

The molecular weights of these polymer samples were estimated from the limiting viscosity numbers $[\eta]$ in specified solvent systems. ${ }^{16,17}$ The contents of ionizable groups were measured by conductometric titrations using a Yanagimoto Conductivity Outfit Model MY-8 after passing through IRA-120B ion exchange column for PVAA, and IRA-410 for Hep. The experimental results are shown in Table $I$. Hep is regarded as an alternating copolymer composed of D-glucuronic acid and D-glucosamine, in which the $\mathrm{SO}_{3} \mathrm{H}$ groups are said to be attached to the $C(6)$ and $C(2)$ of the former and in a

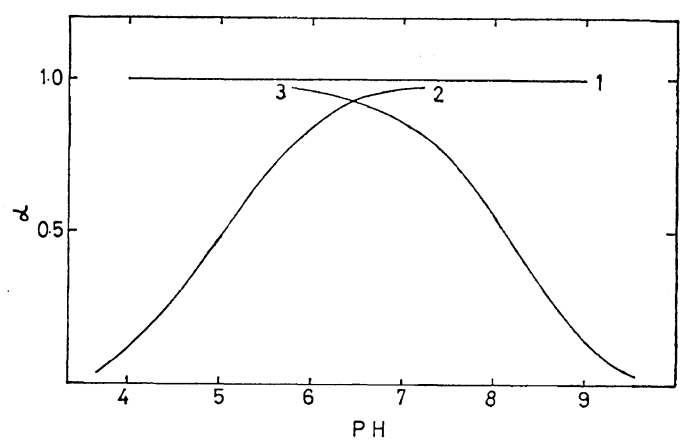

Figure 2. Plots of the degree of dissociation, $\alpha$, against $\mathrm{pH}$ : for $\mathrm{SO}_{3} \mathrm{H}$ groups of $\mathrm{Hep}$, for $\mathrm{COOH}$ groups of Hep, and for $\mathrm{NH}_{2}$ groups of PVAA, designated by curves 1,2 , and 3 , respectively.

small amount to the $\mathrm{C}(2)$ of the latter. The concentration of $\mathrm{COOH}$ groups and $\mathrm{SO}_{3} \mathrm{H}$ groups are $1.70 \times 10^{-3}(\mathrm{eq} / \mathrm{g})$ and $3.36 \times 10^{-3}(\mathrm{eq} / \mathrm{g})$, respectively. Thus the purity of Hep is about $95 \%$ compared with the theoretical value assuming that the structure of Hep is as shown in Figure 1.

The degrees of dissociation of $\mathrm{NH}_{2}$ groups of PVAA and of $\mathrm{COOH}$ groups of Hep were determined by potentiometric titrations. Titrations were performed in the presence of about $0.005-M$ $\mathrm{NaCl}$ under $\mathrm{N}_{2}$ atmosphere for both the polyelectrolyte solution and a reference solution having the same composition but without the polyelectrolyte. The apparatus used for potentiometric titrations was a Hitachi-Horiba $\mathrm{pH}$ Meter Model F-7 $7_{\text {ss. }}$ In Figure 2, the degree of dissociation was plotted against the $\mathrm{pH}$ for $\mathrm{NH}_{2}$ groups of PVAA, and for $\mathrm{COOH}$ groups of Hep. With respect to the $\mathrm{SO}_{3} \mathrm{H}$ groups, the degree of dissociation is unity independent of the $\mathrm{pH}$. The apparent dissociation constants $\mathrm{p} K_{\mathrm{a}}$ were estimated from $\mathrm{pH}$ values at $\alpha=0.5$.

\section{Formation of Polyelectrolyte Complex}

Unless otherwise noted, the polyelectrolytes mentioned above were dissolved at ca. $5 \times 10^{-4} \mathrm{~N}$ concentration in various buffer solutions of varying $\mathrm{pH}$ and ionic strength of ca. 0.005. The polyanion solution at a fixed $\mathrm{pH}$ was then added in different ratios to the polycation solution having the same $\mathrm{pH}$. The quantities of Hep, PVAA, and AO in the system are shown in Figure 3 as an example where $R_{\mathrm{pc}}$ denotes the mixing ratio of polycation. The $\mathrm{pH}$ dif- 


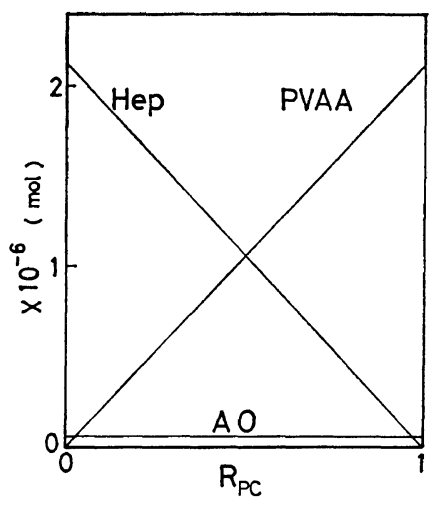

Figure 3. The quantities of Hep, PVAA, and AO contained in the metachromatic reaction system.

ference before and after the addition of polyanion was within 0.03. After formation of the complex, the turbidity and metachromasy of the mixture were measured by a Hitachi Spectrophotometer Model EPS-3T.

The measurement of metachromasy was as follows: After the polyanion solution was added to the polycation solution, any precipitate was removed by centrifuge, and AO was added to the supernatant solution. Then the color change of AO with Hep was measured in the visible spectrum.

The CD spectra were measured at $25 \pm 0.5^{\circ} \mathrm{C}$ by a JASCO J-20 CD/ORD Spectropolarimeter equipped with a quartz cell having a path length 1 to $10 \mathrm{~mm}$. The residue ellipticity, $[\theta]$ in degree $\mathrm{cm}^{2} \mathrm{dmol}^{-1}$, was calculated on the basis of the mole concentration of AO.

Dye concentrations were determined spectrophotometrically using the equation reported by Stone and Bradley. ${ }^{26}$

\section{RESULTS AND DISCUSSION}

\section{Turbidity}

In Figure 4, the turbidity $(\tau)$ of the mixtures is plotted against the mixing ratio $R_{\mathrm{pc}}$ defined as

$$
R_{\mathrm{pc}}=\frac{C_{\mathrm{pc}}}{C_{\mathrm{pc}}+C_{\mathrm{pa}}}
$$

where $C_{\mathrm{pc}}$ and $C_{\mathrm{pa}}$ represent the total numbers of ionizable groups of polycation and polyanion, respectively.

After the mixing process has been carried

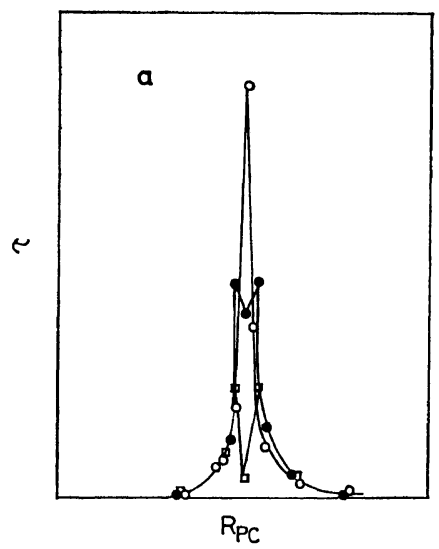

Figure 4a. Time dependence of turbidity in the Hep-PVAA system with various mixing ratios: $\bigcirc$, at $5 \mathrm{~min}$ after mixing; $O$, at $10 \mathrm{~min}$ after mixing; $\square$, at $24 \mathrm{hr}$ after mixing.

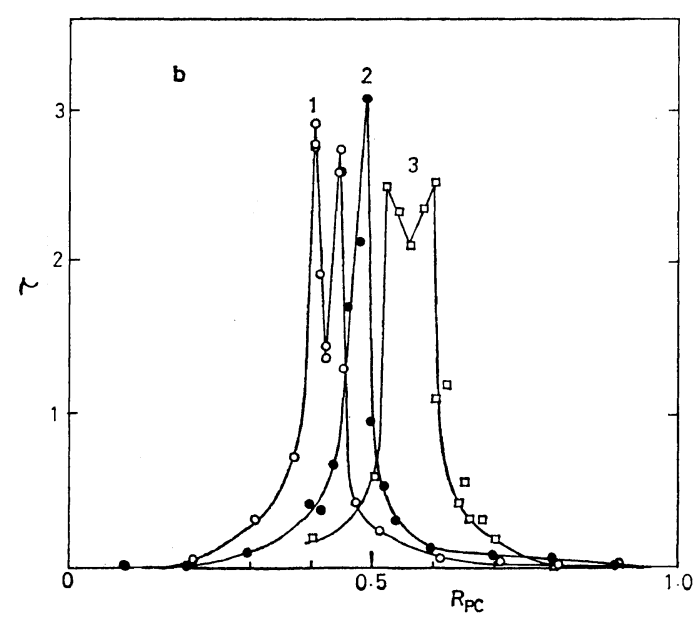

Figure $4 \mathbf{b}$. Turbidity $(\tau) v s$. the mixing ratio $\left(\boldsymbol{R}_{\mathrm{pc}}\right)$ for the Hep-PVAA system at pH 3.20, 4.55 , and 8.45 , shown by the curves 1,2 , and 3 , respectively.

out, the effect of standing time is shown in Figure $4 a$. The maximum in the turbidity curve appeared at an early stage of the standing time and became a minimum as the standing time increased. Such a decrease in turbidity is due to the sedimentation of the complex particles suspended in solution. The yield of polyelectrolyte complex is maximum at the mixing ratio $\bar{R}_{\text {pc }}$ where the turbidity is a maximum or minimum. It was also found that the mixture of Hep and PVAA solutions exists in one of three 
states: (1) in the region where mixing ratio is near $\overline{\boldsymbol{R}}_{\mathrm{pc}}$, insoluble complex particles are unstable and sedimented; (2) in the region where $R_{\mathrm{pc}}$ is greater or smaller than $\overline{\boldsymbol{R}}_{\mathrm{pc}}$ to some extent, insoluble complex particles are stable and dispersed; (3) in the region where $R_{\mathrm{pc}}$ is far from $\bar{R}_{\mathrm{pc}}$, soluble complex particles are formed and the solution is quite clear. The existence of such soluble complex particles will be proved later by metachromasy.

\section{Metachromasy}

The complex formation was investigated also utilizing the property of metachromasy, after the turbidity measurements. Acidic polysaccharides are known to exhibit metachromasy. ${ }^{14}$ Figure 5a shows the metachromasy for AO-

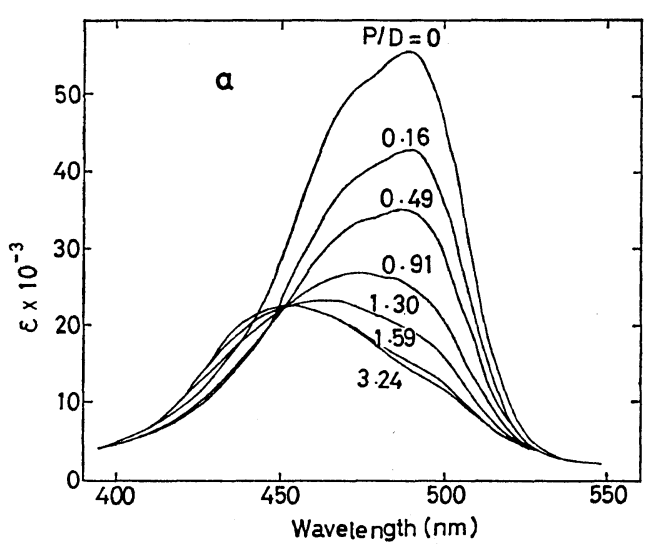

Figure 5a. Metachromatic absorption of AO in Hep under various conditions of $\mathrm{P} / \mathrm{D}$ at $\mathrm{pH}$ 3.3.

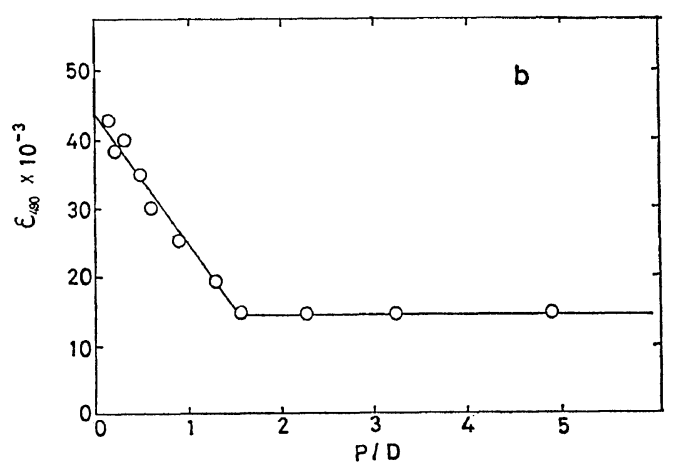

Figure 5b. $P / D$ dependence of molar absorptivity at the free $A O$ absorption band $\left(\varepsilon_{490}\right)$ for HepPVAA system.
Hep system at $\mathrm{pH} 3$ : the $\alpha$-band at $490 \mathrm{~nm}$ originates in the monomeric $\mathrm{AO}$, the $\beta$-band at $470 \mathrm{~nm}$ in the dimeric $\mathrm{AO}$, and the $\gamma$-band in the aggregated $\mathrm{AO}$ on Hep. ${ }^{18}$ It is noted that the height of the $\alpha$-band decreases, but those of the $\beta$ and $\gamma$-bands increase with increasing $\mathrm{P} / \mathrm{D}$ (polymer-to-dye ratio). Hence the value of the $\alpha$-band $\left(\varepsilon_{490}\right)$ can be used as a measure of the monomeric dye (AO) in the solution. This is shown in Figure 5b: the molar absorptivity $\varepsilon_{490}$ of monomeric $\mathrm{AO}$ is plotted against $\mathrm{P} / \mathrm{D}$.

Metachromasy is applied to the investigation of the complex solution. Figure 6a shows the metachromasy for AO-Hep-PVAA system at $\mathrm{pH}$ 3: the $\alpha$-band hardly changes at the mixing

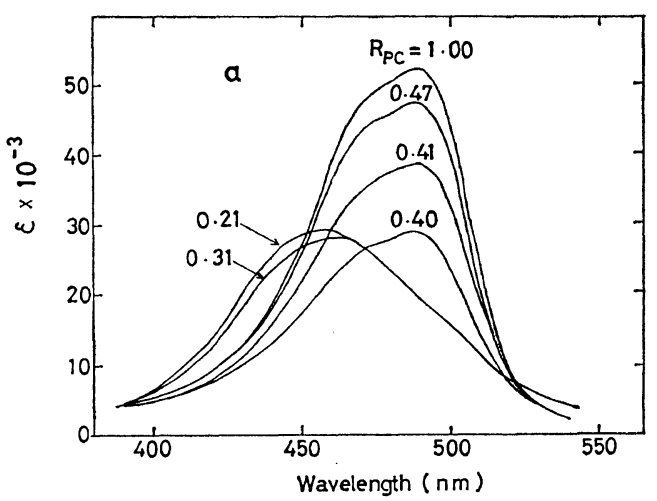

Figure 6a. Metachromatic absorption of $\mathrm{AO}$ in Hep-PVAA system under various conditions of $R_{\text {pe }}$ at pH 3.2.

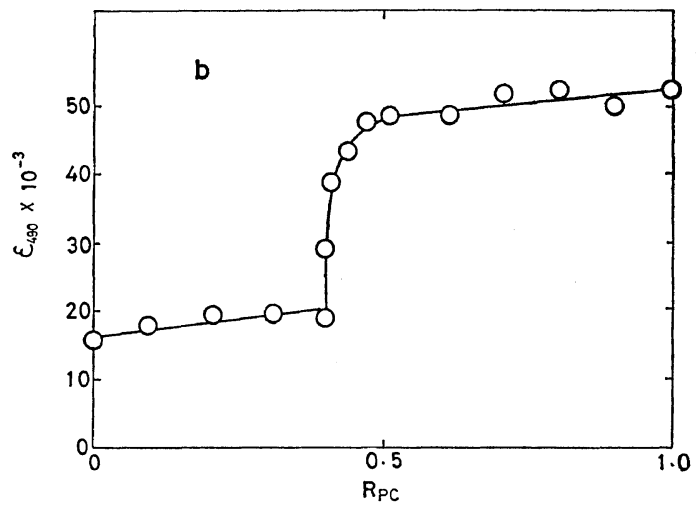

Figure 6b. $R_{\mathrm{pc}}$ dependence of molar absorptivity at the free AO absorption band $\left(\varepsilon_{490}\right)$ for HepPVAA-AO system. 
ratios where $R_{\mathrm{pc}}$ is larger than $\bar{R}_{\mathrm{pc}}$, but changes steeply where $R_{\mathrm{pc}}$ is near $\bar{R}_{\mathrm{pc}}$. Figure $5 \mathrm{~b}$ shows this point more distinctly and some conclusions are derived from Figure $6 \mathrm{~b}$ by comparison with Figure 4b: (1) the value of $R_{\mathrm{pc}}$ where turbidity is a maximum $\left(\bar{R}_{p c}\right)$ is equal to that of $R_{p c}$ where $\varepsilon_{490}$ changes steeply; (2) in the range of $\boldsymbol{R}_{\mathrm{pc}}$ larger than $\overline{\boldsymbol{R}}_{\mathrm{pc}}$, the values of $\varepsilon_{490}$ are almost constant, where Hep does not exist alone in the complex solution. In other words, all the polyanion molecules are incorporated into the complex particles even at the region where turbidity is zero (existence of soluble complex); (3) the value of $\varepsilon_{490}$ changes to some extent in a range of $R_{\mathrm{pc}}$ being somewhat larger than $\bar{R}_{\mathrm{pc}}$. This seems to indicate the existence of Hep anionic groups which do not participate in the complex formation but are in the complex particle.

\section{Stoichiometry}

$\bar{R}_{\mathrm{pc}}$ thus obtained by the measurements of turbidity and metachromasy is plotted against $\mathrm{pH}$ in Figure 7. If the reaction between both components is stoichiometric, then a perfectly neutral complex is formed at $\bar{R}_{\mathrm{pc}}$, and the following equation should hold

$$
C_{\left(\mathrm{SO}_{3} \mathrm{H}\right)}+\alpha C_{(\mathrm{COOH})}=\beta C_{\left(\mathrm{NH}_{2}\right)}
$$

where $\alpha$ and $\beta$ represent the degrees of dissociation of the COOH groups of Hep, and of $\mathrm{NH}_{2}$ groups of PVAA, respectively, at given $\mathrm{pH}$ values, and are obtained from the curves in Figure 2. $C_{\left(\mathrm{SO}_{3} \mathrm{H}\right)}, C_{(\mathrm{COOH})}$, and $C_{\left(\mathrm{NH}_{2}\right)}$ represent the numbers of ionizable groups, $\mathrm{SO}_{3} \mathrm{H}, \mathrm{COOH}$, and $\mathrm{NH}_{2}$, respectively. Since $C_{\left(\mathrm{SO}_{3} \mathrm{H}\right)}=2 C_{(\mathrm{COOH})}$ (see Table I), the composition $\left(\bar{R}_{\mathrm{pc}}\right)$ of the complex formed by stoichiometric reaction is obtained from the following equation.

$$
\begin{aligned}
\bar{R}_{\mathrm{pc}} & =\frac{C_{\mathrm{pc}}}{C_{\mathrm{pa}}+C_{\mathrm{pc}}}=\frac{C_{\left(\mathrm{NH}_{2}\right)}}{C_{\left(\mathrm{SO}_{3} \mathrm{H}\right)}+C_{(\mathrm{COOH})}+C_{\left(\mathrm{NH}_{2}\right)}} \\
& =\frac{C_{\left(\mathrm{NH}_{2}\right)}}{3 C_{(\mathrm{COOH})}+C_{\left(\mathrm{NH}_{2}\right)}}=\frac{\alpha+2}{\alpha+3 \beta+2}
\end{aligned}
$$

This curve obtained assuming the reaction to be stoichiometric is also shown in Figure 7.

As is obvious from Figure 7, the reaction in the Hep-PVAA system is not perfectly stoichiometric as reported for system of diethoxyethyl trimethyl ammonium PVA-sulfated PVA. ${ }^{19}$ The

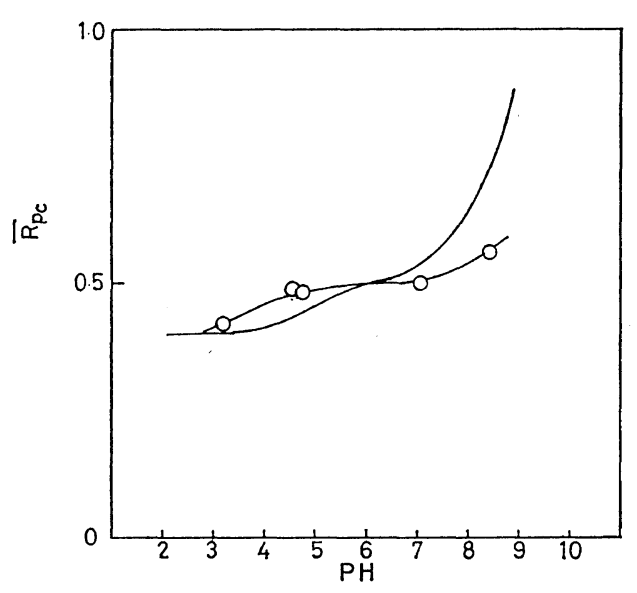

Figure 7. Compositions of complex $\left(\bar{R}_{\mathrm{pc}}\right)$ are plotted against $\mathrm{pH}$ for Hep-PVAA system.

discrepancy between both curves may be attributed to two reasons: (1) un-ionized groups are induced to ionize by ionized groups with opposite signs, thus $\alpha$ and $\beta$, respectively, increase at lower and higher $\mathrm{pH}$ regions, compared with the degree of ionization of the corresponding polyelectrolytes in the absense of a partner polyelectrolyte; (2) Hep consists of pyranose rings shown in Figure 1, and is more rigid compared with the polyelectrolyte having flexible chains such as vinyl polymer, thus oneto-one correspondence seems difficult between oppositely charged groups of polyanion and polycation molecules owing to the difficulty of changing the interionic group distance. The mean interionic group distance of $\mathrm{Hep}$ is $15.9 / 6=2.65(\AA)$ from the fiber period of the X-ray diffraction, ${ }^{27}$ while that of PVAA is $2.5 \times 1000 / 7.5=33.3(\AA)$ when fully stretched, since the main chain of PVAA is made from $-\mathrm{CH}_{2}-$ unit. It is evident that the distance of interionizable groups of the fully stretched PVAA, i.e., $33 \AA$, is difficult to contract to $2.7 \AA$. But the discrepancy between both curves is not as great for Hep-PVAA system as it is for the system containing cellulose derivatives such as sulfated cellulose and carboxymethyl cellulose. ${ }^{4}$ This is probably due to the fact that the backbone chain of polysaccharide containing $\alpha-1,4-$ linkage is more flexible than that containing $\beta$-1,4-linkage. 


\section{Induced $C D$}

Induced $C D$ is a useful means for investigating the conformation of the polymers which have no absorption band above $200 \mathrm{~nm}$. This technique is applied to the study of Hep which is in the complex particles. The induced $C D$ spectra of Hep-AO system are shown in Figure 8. This figure may indicate that Hep takes a helical conformation even locally in the solution. This is consistent with the results on the methylene blue-heparin system as indicated by the induced $C D$ measurement and on the Hep by farultraviolet $\mathrm{CD}^{20,21}$ The bands at $425 \mathrm{~nm}$ and $450 \mathrm{~nm}$ at $\mathrm{pH} \mathrm{3,} \mathrm{together} \mathrm{with}$ those at $435 \mathrm{~nm}$ and $480 \mathrm{~nm}$ at $\mathrm{pH} 8$, may be responsible for the electronic transitions polarized perpendicular and parallel, respectively, to the axis of the helix, if the result on the poly(glutamic acid)-AO system is taken into consideration. $^{22,23}$ Also the band at $480 \mathrm{~nm}$ at $\mathrm{pH} 3$ may responsible for dimeric AO. From these results it is concluded that Hep takes a left-handed helical structure, because the electronic transition polarized parallel to the helix axis is negative. The induced $C D$ does not always reflect the conformation of native polymer: for example a characteristic absorption spectrum is observed for the AO-poly(glutamic acid) system at a higher $\mathrm{pH}$, where poly(glutamic acid) takes on a random coil conformation. ${ }^{24,25}$ But when $\mathrm{P} / \mathrm{D}$ is lager than 1 , no characteristic CD spectrum is observed. Thus all experiments were performed at $\mathrm{P} / \mathrm{D}$ larger than 1 . The difference between the spectra at $\mathrm{pH} 3$ and $\mathrm{pH} 8$ is not clear from just these data. But according to Stone, Hep takes a more ordered structure (helix) at $\mathrm{pH} 3$ than at $\mathrm{pH} 8$, so the difference between these spectra may be due to the change in conformation accompanying the change in the $\mathrm{pH}$.

The CD spectra of Hep-PVAA-AO system is shown in Figure 9. As described above, ${ }^{11}$ AO should be expected to bind to the anionic groups in the complex, but not have a decisive influence on the structure of the complex. So the bound AO may reflect the conformation of Hep in the complex. As is the case in Figure 8 , it is concluded that Hep takes a left-handed helical conformation in the complex. It is worth noting that the spectrum of Hep-PVAA-AO system at $\mathrm{pH} 3$ is close to that of Hep-AO system at $\mathrm{pH} 8$ rather than that of Hep-AO system at $\mathrm{pH} 3$. This is probably because the helical (ordered) structure of Hep is broken by the formation of the complex with PVAA, if we assume that the change in spectra with $\mathrm{pH}$ for Hep-AO system is due to the conformational change as mentioned above.

Another explanation of the induced $C D$ for AO-poly(glutamic acid) system has been presented recently by Ikeda, et al. ${ }^{25}$ in which the assignment for each band or the sign of helical sense is different from those mentioned above. These points should be discussed futher in detail. However, it is evident that at a P/D

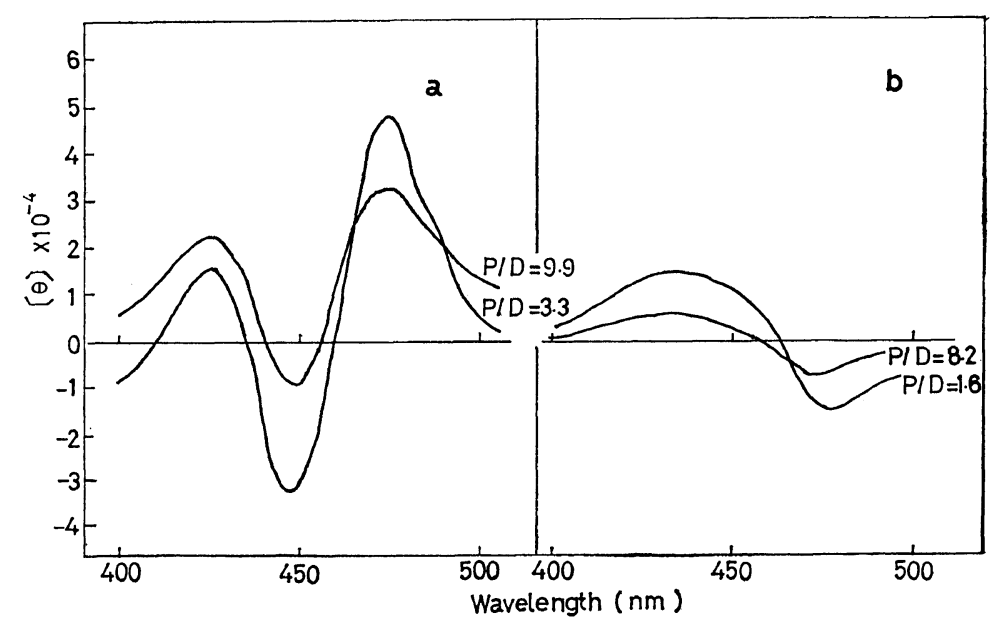

Figure 8. CD spectra of Hep-AO systems (molar ellipticity vs. wavelength) at (a) $\mathrm{pH} 3$ and (b) $\mathrm{pH} 8$. 


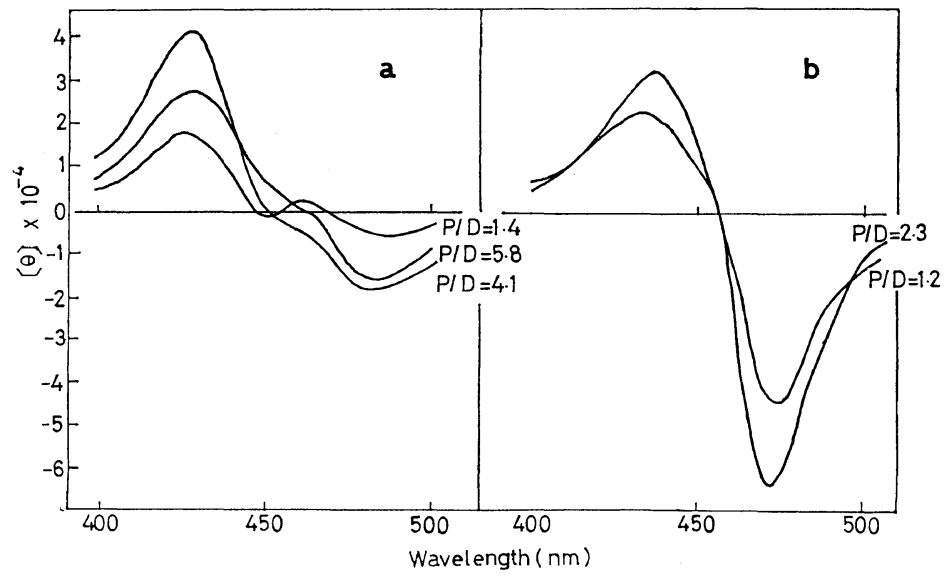

Figure 9. CD spectra of Hep-PVAA-AO systems at (a) $\mathrm{pH} 3$ and (b) $\mathrm{pH} 8$.

larger than 1 , the induced $C D$ reflects an ordered structure of the native polymer, and so this can be used as a means of studying the conformation of the polymers.

We have discussed the properties of ionizable groups, i.e., $\mathrm{COOH}, \mathrm{SO}_{3} \mathrm{H}$, and $\mathrm{NH}_{2}$ groups, and the electrostatic interaction between those groups. Another interaction (particularly the hydrogen bond) is possible to some extent between Hep and PVAA according to the structures shown in Figure 1. However Hep and PVAA react primarily through the electrostatic interaction to bring about precipitation accompanied with the loss of charges, and so a nonelectrostatic interaction is not very important. The effect of the hydrogen bond cannot be separated from that of electrostatic interaction at present.

The above mentioned results will be further examined by light scattering measurements. The authors acknowledge their sincere appreciation to Drs. K. Shinoda, H. Sato, and T. Hayashi for helpful comments.

\section{REFERENCES}

1. E. S. Wajnerman, W. Ja. Grinberg, and W. B. Tolstogusow, Kolloid Z. Z. Polym., 250, 945 (1972).

2. M. Hosono, O. Kusudo, S. Sugii, and W. Tsuji, Bull. Inst. Chem. Res., Kyoto Univ., 52, 442 (1974).

3. A. Nakajima and K. Shinoda, J. Colloid Interface Sci., 55, 126 (1976).
4. H. Sato and A. Nakajima, Polym. J., 7, 241 (1975).

5. R. A. Gelman, W. B. Rippon, and J. Blackwell, Biopolymers, 12, 54 (1973).

6. R. A. Gelman, D. N. Glaser, and J. Blackwell, ibid., 12, 1223 (1973).

7. R. A. Gelman and J. Blackwell, ibid., 12, 1959 (1973).

8. R. A. Gelman and J. Blackwell, ibid., 13, 139 (1974).

9. A. Nakajima, K. Shinoda, T. Hayashi, and H. Sato, Polym. J., 7, 550 (1975).

10. K. Shinoda, K. Sakai, T. Hayashi, and A. Nakajima, ibid., 8, 208 (1976).

11. K. Shinoda, T. Hayashi, T. Yoshida, K. Sakai, and A. Nakajima, ibid., 8, 202 (1976).

12. K. Shinoda, T. Hayashi, and A. Nakajima, ibid., 8, 216 (1976).

13. H. Sato, T. Hayashi, and A. Nakajima, ibid., 8, 517 (1976).

14. A. L. Stone, "Structure and Stability of Biological Macromolecules," Marcel Dekker Inc., New York, N.Y., 1969, Chapter 5, p 353.

15. B. C. Myhr and J. G. Foss, Biopolymers, 10, 425 (1971).

16. P. A. Liberti and S. S. Stivala, Arch. Biochem. Biophys., 119, 510 (1967).

17. A. Nakajima and K. Furutachi, Kobunshi Kagaku (Chem. High Polymers), 6, 460 (1948).

18. D. F. Bradley and M. K. Wolf, Proc. Natl. Acad. Sci. U.S.A., 45, 944 (1959).

19. M. Hosono, O. Kusudo, S. Sugii, and W. Tsuji, Report of the Poval Committee, Kyoto, 61, 79 (1972).

20. A. L. Stone and H. Moss, Biochim. Biophys. Acta, 136, 56 (1967). 


\section{Hara and A. Nakajima}

21. A. L. Stone, Biopolymers, 7, 173 (1969).

22. R. E. Ballard, A. J. Mccaffery, and S. F. Mason, Biopolymers, 4, 97 (1966).

23. M. Hatano, M. Yoneyama, and Y. Sato, Biopolymers, 12, 895 (1973).

24. Y. Sato and M. Hatano, Bull. Chem. Soc. Jpn., 46, 3339 (1973).
25. T. Imae and S. Ikeda, Biopolymers, 15, 1655 (1976).

26. A. L. Stone and D. F. Bradley, J. Am. Chem. Soc., 83, 3627 (1960).

27. I. A. Nieduszynski and E. D. T. Atkins, Biochemistry, 135, 729 (1973). 